\title{
B-Mode Ultrasound Diagnosis in Ocular Trauma and its Accuracy
}

\author{
Nazeeha Waseem ${ }^{1 *}$, Saman Sanaullah ${ }^{2}$, Rehan Afsar ${ }^{3}$, Muhammad Ahmad Naeem ${ }^{4}$, Abid Ali $^{5}$
} ${ }^{1,2}$ Medical Imaging Doctor, Department of Radiological Sciences and Medical Imaging, the University of Lahore, Gujrat, Pakistan
${ }^{3,4}$ Lecturer, Department of Radiological Sciences and Medical Imaging, the University of Lahore, Gujrat, Pakistan
${ }^{5}$ Associate Professor, Department of Allied Health Sciences, the University of Lahore, Gujrat, Pakistan

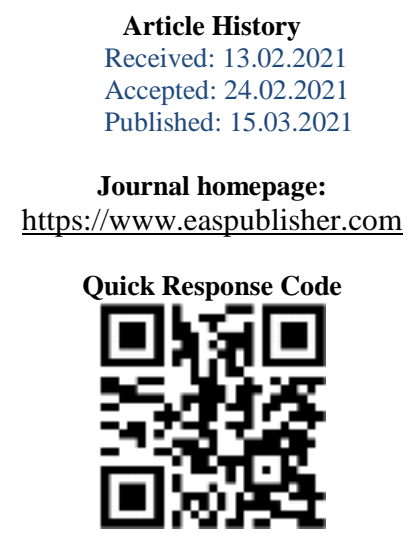

\begin{abstract}
The incidence of ocular trauma has been on the rise for the past few years, which urges the medical community to be more apt at diagnosing and treating this condition. An effective way to diagnose different etiologies of ocular trauma involves the use of ultrasound to visualize the anatomical layers of the eye and orbit, which is vital for deciding the best management plan. In this review article, the discussion will be about visual injury, the function of ultrasound in diagnosing this condition, and the accuracy of B-mode ultrasound. The gathered data and all information will assistance of physicians become more effective in the managing Ocular trauma.

Keywords: ocular trauma; B-mode; vector site.
\end{abstract}

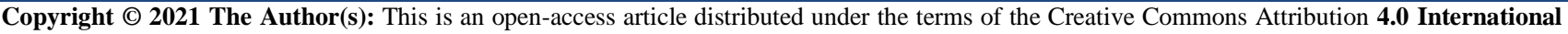
License (CC BY-NC 4.0) which permits unrestricted use, distribution, and reproduction in any medium for non-commercial use provided the original author and source are credited.
\end{abstract}

\section{INTRODUCTION \\ Ocular trauma}

Ocular trauma is defined as a disorder, linked with the blindness and visual impairment. Globally, fifty-five millions fresh cases of ocular trauma are being reported annually, having worse conditions including irreversible blindness. To minimize the degree of tissue damage and after investigating the condition of the patient surgery would consider as a final way out to this situation. The advancement in the diagnosis of the disease required expert physicians having command on the conceptual basis of ocular trauma. The following paper will assist the reader widely in coping with the discussion of ocular trauma, Ultrasound diagnosing of this disease and will also enlighten the B-mode ultrasound and its accuracy.

\section{Epidemiology of Ocular Trauma}

\section{Yearly frequency of OT}

It's been really tough to get data that is comprehensive related to the frequency of ocular trauma because of less reliable sources. Globally, various investigations done by institutions and healthcare facilities for checking the yearly frequency of ocular trauma in patients admitted to hospitals already. As per statics in United States the OT cases ranged from 8.2 to 13.0 per 1000 people between the years 1992-2001 [1]. This comes out to be about 2 million reported cases in this timespan.

Following are the regions of the world where OT is reported:

- $\quad$ Singapore - 12.6 [2]

- Australia (old above 40) - 21 [3]

- $\quad$ Singapore - 12.6 [4]

Reports from the Wang's study says that almost, patients admitted already in hospitals have accounted by OT about $1 / 3$ of eye disorder [5].

\section{At-risk individuals}

Majorly cases of seriously ill reported about 19 million annually along with the 55 million mild cases of OT. Usually more cases have been reported in males having ocular trauma relative to other gender generally with the ratio of $4: 1$ [6]. Besides this older adult have more high risk of vision impairment and hence it's a major factor in poor diagnosis of case if reported. So, age is an important factor in this regard. But it also prone to younger individuals of age 10 or less and they have risk of getting OT, because at the said age of 10 one can sustain about $80 \%$ higher risk of OT. Other factors include: 
- Lack of quality education

- Engaging in hectic and tough labor workings

- Not well socioeconomic status

\section{Sources of Ocular trauma Workplace mostly renders to the source of Ocular trauma}

More common sources of ocular trauma are related to workplace and is being reported in the collective research based in Singapore and China [7]. Besides this road accidents are also some of the sources of OT. Professionals identified that commonly blunt trauma and foreign bodies are the sources of injury. Wood branches, rocks, professional instruments, sticks, glass and fist or feet reported as primary reason of the ocular trauma in America and China

Below are some things that may cause the Ocular trauma:

- Writing pens

- Glass

- Nails

- Metallic objects

Sometimes physical activities including sports (Baseball, Volleyball etc.) can be the reason of OT [9]. Due to the diversity in the nature of the causes of the ocular trauma risk factor at population level is high. Hence it is really the need to work on the cure and putting efforts in managing this condition to minimize risk factors of OT accordingly. Now the next section will discuss in detail about Ultrasound usage in OT cure and diagnosis along with B-mode ultrasound and its accuracy.

\section{Diagnosis of Ocular trauma using ultrasound}

In B-mode arrangements both transducer scanners and signal processing are used in order to get acoustic images of various ocular structures. And its quality is reliant on the factors similar to A-mode arrangements [19]. Other things that can't be neglected in quality identification are electronic, mechanical, and acoustic elements. Moreover, B-mode can be used for getting various artifacts which can be identified with ease and then may remove [22]. While working B-mode system get benefited itself by liner transducer and the transducer generates ultrasonic pulses. These pulses reflect to the device with equal intervals i.e. spaced with no difference in positions [23].

\section{These equally spaced positions taken as vectors [24]}

During the manufacturing of the ultrasound machines it is kept in mind that the spacing between vectors should be not more than transducer beam-width in the principal place. But in case the spaces are large then to see all anatomical structures vectors will also be sparse.

As scan motion is not discontinued so each vector site is noticed individually. Just like A-mode systems echo signals are treated in the similar patterns [25]. The signals will be dealt in such a way that to control the display intensity before finalizing the video to get graph between echoes amplitude verses range. Scan converters used to facilitate the processes, in which every pixel constructed is displayed [26]. There is simplicity when we deal with the line scan wave as the relationship among the locations and signal envelope is not complicated. Every axis is correlated to a range while considering the scan converter, along with transducer position having suitable setting of the scales. To occupy the free memory locations interpolation is carried out as scan converter has many lines as compared to vectors. So, screen shows tissue mirror image in respect to radiance as variable of interval or distance from the transduction, pixels specify $2 \mathrm{D}$ location of reflective faces in the scrutinize matter.

The pictures that are produced are wellseparated borders (e.g. the precedent lens surface) that make a described as A-mode resonances and displayed by way of separate planes. Acoustically identical sections are not shown as bright parts, while heterogeneous zones will be seen as congruently spotted illumination or not darker designs. Supposition, B-mode picture production comprises of non-variable pace of sound number during the complete photo facilitating, and the formation of photo by permitting assimilation of a one scale factor to produce geometrically precise photo [27]. But, pace of sound is variable all over the picture and doesn't remain constant when penetrating various ocular tissues. The parts of eye including cornea, sclera and certain tumors have high sound speed in contrast to vitreous. Variance remains as a core of anamorphic alteration, known as key factor in determining inaccuracies in Bio-metric findings. Tissues depth of ocular and the location of the vectors are kept in mind while doing anamorphic correction of the biometry.

\section{Different scan designs used by B-mode arrangements [28]}

Commonly and also best designs can generate ultrasonic beams with vertical arrangement along reflective tissue planes. With the usage of these designs, echoes will reflect to the transducer in undeviating patterns. Linear image arrangements attain vertical patterns over minor parts of curved ocular surfaces, i.e. retina. Therefore, images that are displayed are not showing full eye. In comparison to this, sector scan designs work well in dealing bent planes, and hence Bmode's posterior planes are to be captured by echoes. Arc scan designs permit the vertical placement over the frontal planes of OT structures and lens. Besides the designs we have discussed, in ophthalmology commonly used design is sector design. To permit the contact ocular inspection the sector scan has been applied in such a way that it has some sealed footprints having compact probe. Furthermore, the screening examination comprises the physical indication of the 
transducer results in attaining high scan duplication rates. The study of concentration method and lid speculum play vital role in transducer occurrence nearly forty mega Hertz. Arc scan can be utilized as it is better in envision of the cornea and other anterior structures but cannot be done in contact inspections.

\section{B-mode image quality}

In ideal case of B-mode images pixel intensity will resemble with every part of tissue during ultrasound reflectivity. But there are some limitations i.e. intensity ranges of monitors, video printers will not allow this to occur. 1 Byte RGB is provided by modern digital devices and hence permitting for maximum display of the millions of colors at once having limitation set at two fifty-six levels of every shade. Example of it is, gray color mainly comprised of pixels sharing intensity range of RGB. So, two fifty-six shades of gray showed which resembles to a Twenty-four-dB dynamic range. Though when we accelerate the effective $\mathrm{dB}$ applying basic logarithmic amplifications, then more opportunity is to practice the usage of Bmode pictures in order to get the assessment of general anatomy though finding A-mode outcomes regarding quantitative reflectivity data. Three-dimensional quality related to B-mode arrangement, restricted by pixel firmness and showing-tool, along with ultrasound limitations (for example: aperture, focal length and frequency).

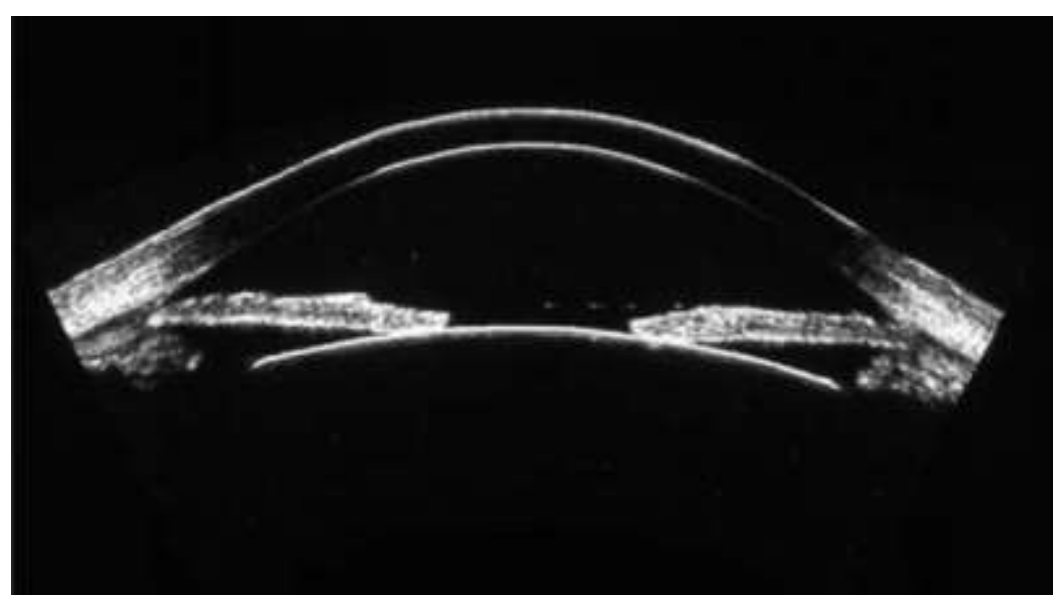

Fig-1: High-frequency (50 MHz) scan of anterior segment produced using arc-scan geometry. This scan geometry maintains near-normality relative to the anterior surfaces of the globe, allowing display of the full corneal contour [31]

\section{Real-time imaging}

While operating B-mode, pictures showed that at the equivalent rate the quantity of scans ran by the analysis. Mechanical segment scanner offers 4 scans/second, but modern tool can provide scans at thirty Hertz or greater than it [20]. The actual valuation of OT assemblies is credible and of high rank in evaluation of translucent tissues, retinal displacement, intraocular bleeding. Additionally, actual feature or real-time is helpful in envisioning the vascular plasticity regarding tumors and, hence aiding fixed assessment [21]. Actual inspection, specialists, surgeons can practice cine-loop by fastening camera parallel to output of B-mode.

\section{Artifacts of B mode}

Pictures produced using B-mode then disposed to artifacts where they initiate from acoustic and electronic foundations. Artifacts, outcome gets directly by dissimilarity of transmission pace during the course of various OT assemblies [29]. Beside an essential path ophthalmic artery(OA) done by lens, back seems as it moved anteriorly since the high lenticular velocity reductions from the point $A$ to the transducer keeping in view of transit time. Additionally, scan paths crossing indirectly from lens subject the ultrasound pulse to refraction so the point that is being imaged does not lie along the transducer pivot. In contrast to this, paths sidestepping the lens outcome in exact imaging. Total consequences by these methods are just altering reliefs of tissues positioned posterior to lens. Alternative kind of artifact, acoustic shadowing, is associated to the tissue region posterior to high absorptive parts where minimization of light intensity happens including lens and some parts of tumors. Differential diagnosis is mostly assisted by shadowing as it permits the physician to sort out the tumors depending on the level of absorptivity. As outcome, accurate findings obtained because now the transducers won't transverse the lens. Sensibly leaning the scans over the sclera gives the negligible deprivations from velocity and other absorption effects. Numerous acoustic reflections establish additional basis of artifacts, presenting repetition of muscular lines. Absorption scan, ultrasonic echoes return to transducer from the cornea and lens implant and are not fully bounced directed to eye. When the transducer-cornea separation determines the transit time the echoes returned to the transducer second time as being reflected by the cornea.

The large number of mirrored reverberations seems mutually in a mode signals and B-mode pictures, and then it typically seen as phantom planes inside vitreous or in rare portions. Acknowledgment of various 
reflections is straightforward: varying the transducercornea standoff distance results in changing of the position of the artifacts with the respective motion observed in some parts of the eye. All artifacts easily removed if one can start creating this standoff distance identical to depth of tissue at maximum while examining. Reduplication artifacts similarly happen in contact scans, although uncommon. Electronic artifacts adopt numerous procedures. "Snow 'can show up on Bmode images, if intensifier gain is high and electronic clamor isn't dismissed preceding display. Orbital fat, a heterogeneous structure, can be seen as bright areas uniformly due to saturation. Recognition of the said artifacts is assisted by cautious checking of A-mode indicators. Digital storage of B-mode pictures discusses numerous benefits in postprocessing. Enhancement methods are applicable to the image with the help of digital representation and hence it is linked directly with the application of numerous digital image processing. Most tools comprise a set of non-complicated procedures, such as brightness, contrast adjustment, and, possibly, a zoom function.

Related to digital image enhancement (3D), literature is available on a large scale. One can apply it to generic format of ultrasonic $\mathrm{B}$-mode images including TIFF or JPEG. Many operations that are benefited in terms of thee modification of the median filtering, thresholding, intensity curve and brightness of pixels etc. Such processes can be easily done using accessible software, including NIH-Image or Photoshop. Additionally, a shade gauge may be replaced for the typical grayscale in expressing pixel glare. Using of shades (colors) offers amplified contrast in contrast with the grayscale. The default screen mode will show colorful screen when we see its display mode in the optical coherence tomography, e.g. While using B-mode ultrasound imaging one can't see colorful screen as it is not achievable in this, the reason behind this is arbitrary nature of the color scale as they exposed in non-judiciously environment.

\section{B-mode ultrasound accuracy in OT}

Prior it is mentioned, initial analysis and involvement may be the key stages to avoid visual impairment and other vision problems in patients experiencing visual injury. Ultrasound B-scan easily recognized as remarkably comprehensive $2 \mathrm{D}$ pictures of the cornea, anterior sclera, aqueous cavity (chamber), and posterior chamber [10]. Prior to operation, surgeon should check extent of interior imbalances and should see that which things they will encounter. B-scan showed to be the faultless instrument that will provide advantageous data about the below listed basic grievances:

- Vitreous hemorrhage

- Discoloration of lens

- Ruptured lens

- Coats having detachments

- Foreign bodies presence

\section{- Globe rupture}

For the serious patient of visual injury, microsurgery is the proposed technique to address the irregularities. This implies that pre-employable ultrasound appraisal is the best way to assess and design the process [11]. Different researchers have expressed that by ultrasound procedure we can distinguish different kinds of visual injury in a wide pool of patients. Vrablki ME showed that the discovery capacity of ultrasound while detecting retinal displacement range between $97 \%-100 \%$ and $83 \%-100 \%$ individually [12]. Likewise, these components arrived at an affectability of $84.6 \%$ and a particularity of $98.3 \%$ for lens separation [13]. To know the accuracy of Bmode ultrasound, we should to examine the pathophysiology and how it considers acoustic imaging. During visual injury, visual media gets obscure because of blood collection and hemorrhages, and hence leads to development of the posterior segment difficult.

So, visualization of the fundus affected because in a few hours traumatic cataract would develop. When someone is at this condition, as examining the eye is not easy so the diagnosis of ocular trauma is obstructed. But due to available technique like ultrasound B scan the diagnosis of ocular trauma that is directly linked with muddiness of transparent material from where light passes. With the help of technique one can be benefited regarding medical care offices and crisis divisions that manage a high rate of visual injury consistently. In one investigation directed in the city of Karachi, ultrasound B check end up being extremely compelling in perceiving and identifying ocular trauma. Ultrasound B scan showed that when 8 cases were examined using it 5 reported the accurate agreement with the surgical diagnosis. As for the 3 other cases, the study showed a very good agreement. There is conflicting theory among various users and setting regarding operator dependency of ultrasound but there are a lot of reports cited globally that showing alike outcomes.

It is stated by Kim S et al as mentioned below: $73 \%$ sensitive and specificity of about $90 \%$ It is stated by Imran $\mathrm{S}$ et al as mentioned below: $84.6 \%$ sensitive and specificity of about $96.5 \%$ in order to spot vitreous hemorrhage [15].

Back to the study of Karachi city, which additionally demonstrated that ultrasound $\mathrm{B}$ scan has a sensitivity of $100 \%$ and a specificity of $97 \%$ when it comes to detecting foreign body presence in ocular trauma. Most types of intraocular foreign bodies comprising metals and other materials $93 \%$ sensitivity of ultrasound is reported by one of the studies, Amanullah et al. [16]. In the ED, for detecting victims of ocular trauma ultrasound is best suitable mainly for the countries where proper surgical equipment is not easily assessable. Indeed, the accuracy of B-mode 
ultrasound isn't like that of CT check [17], which is a startling end because of the cost and working expense of CT filters. Besides, the perceptible points of interest of B-mode ultrasound, the availability and convenience of this gadget make it a hugely viable gadget in every clinical setting, explicitly those swarmed by injury patients, for example, careful focuses and crisis divisions. Furthermore, studies displayed a considerable decrease in the mean assessment time for ocular trauma irrespective of patients' turnover [18]. Patients having complaints about posterior segment ultrasound B scan is suggested as preliminary investigating purposes but with monotonous repetition in ocular injuries.

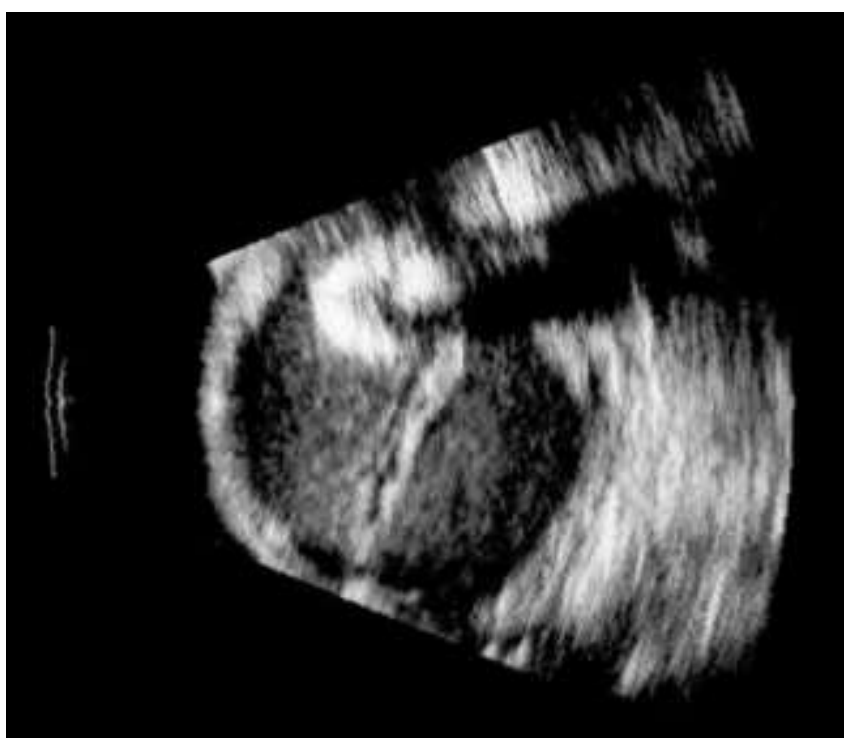

Fig-2: Displaced hypermature cataractous lens in eye through vitreous hemorrhage and whole retinal dispassion. Lens material is highly acoustically absorptive, resulting in an acoustic shadow trailing from the lens.[32]

\section{CONCLUSION}

In outline, ongoing ultrasound B-filter is an essential dependence on the actual assessment of visual injury related with an opaque media. In cases in which medical procedure isn't thought of, ultrasound is valuable as a subsequent assessment to survey goal and wipe out the improvement of retinal separation. Visual injury is a typical boss disease seen in emergency clinics and private facilities. This issue conveys a significant peril of vision hindrance and lasting visual deficiency. Thus, it is crude for doctors to dominate the utilization of ultrasound in diagnosing this condition to quicken the remedial strategy and reduce the danger of long lasting sequela. The mechanism, accuracy and detection of the ocular trauma in various conditions is reviewed by considering the B-mode ultrasound system. We also cited the scientific papers and clinical studies that support the statements made. Hopefully, the gathered data and all information will assistance of physicians become more effective in the managing Ocular trauma.

\section{REFERENCES}

1. McGwin, G., Hall, T. A., Xie, A., \& Owsley, C. (2006). Trends in eye injury in the United States, 1992-2001. Investigative ophthalmology \& visual science, 47(2), 521-527.

2. Desai, P., MacEwen, C. J., Baines, P., \& Minassian, D. C. (1996). Epidemiology and implications of ocular trauma admitted to hospital in Scotland. Journal of Epidemiology \& Community Health, 50(4), 436-441.

3. Voon, L. W., See, J., \& Wong, T. Y. (2001). The epidemiology of ocular trauma in Singapore: perspective from the emergency service of a large tertiary hospital. Eye, 15(1), 75-81.

4. McCarty, C. A., Fu, C. L., \& Taylor, H. R. (1999). Epidemiology of ocular trauma in Australia. Ophthalmology, 106(9), 1847-1852.

5. Wang, B., Congdon, N., Bourne, R., Li, Y., Cao, K., Zhao, A., ... \& Wang, N. (2018). Burden of vision loss associated with eye disease in China 1990-2020: findings from the Global Burden of Disease Study 2015. British Journal of Ophthalmology, 102(2), 220-224.

6. Xu, J. F., \& Wang, Y. (2004). Epidemiology of eye injury in mainland of China. Int $J$ Ophthalmol, 4, 1069-1076.

7. Wong, T. Y., Klein, B. E. K., Klein, R., \& Tomany, S. C. (2002). Relation of ocular trauma to cortical, nuclear, and posterior subcapsular cataracts: the Beaver Dam Eye Study. British journal of ophthalmology, 86(2), 152-155.

8. Wong, T. Y., Klein, B. E., \& Klein, R. (2000). The prevalence and 5-year incidence of ocular trauma: the Beaver Dam Eye Study. Ophthalmology, 107(12), 2196-2202.

9. Krishnaiah, S., Nirmalan, P. K., Shamanna, B. R., Srinivas, M., Rao, G. N., \& Thomas, R. (2006). Ocular trauma in a rural population of southern 
India: the Andhra Pradesh Eye Disease Study. Ophthalmology, 113(7), 1159-1164.

10. Vrablik, M. E., Snead, G. R., Minnigan, H. J., Kirschner, J. M., Emmett, T. W., \& Seupaul, R. A. (2015). The diagnostic accuracy of bedside ocular ultrasonography for the diagnosis of retinal detachment: a systematic review and metaanalysis. Annals of emergency medicine, 65(2), 199-203.

11. Sawyer, M. N. A. (2009). Ultrasound imaging of penetrating ocular trauma. The Journal of emergency medicine, 36(2), 181-182.

12. Vrablik, M. E., Snead, G. R., Minnigan, H. J., Kirschner, J. M., Emmett, T. W., \& Seupaul, R. A. (2015). The diagnostic accuracy of bedside ocular ultrasonography for the diagnosis of retinal detachment: a systematic review and metaanalysis. Annals of emergency medicine, 65(2), 199-203.

13. Haghighi, S. H. O., Begi, H. R. M., Sorkhabi, R., Tarzamani, M. K., Zonouz, G. K., Mikaeilpour, A., \& Rahmani, F. (2014). Diagnostic accuracy of ultrasound in detection of traumatic lens dislocation. Emergency, 2(3), 121.

14. Kim, S., \& Lee, S. (2005). Comparison of ultrasound and intraoperative finding in patients with vitreous hemorrhage. Investigative Ophthalmology \& Visual Science, 46(13), 54365436.

15. Imran, S., Amin, S., \& Daula, M. I. H. (2011). Imaging in Ocular Trauma: Optimizing the Use of Ultrasound and Computerised Tomography. Pakistan Journal of Ophthalmology, 27(3).

16. Ullah, A., Khan, B. S., Alam, M., \& Khan, M. D. (2006). Ambulatory cataract surgery IN public health sector in NWFP: A study of 302 cases. Journal of Postgraduate Medical Institute (Peshawar-Pakistan), 20(3).

17. Imran, S., Amin, S., \& Daula, M. I. H. (2011). Imaging in Ocular Trauma: Optimizing the Use of Ultrasound and Computerised Tomography. Pakistan Journal of Ophthalmology, 27(3).

18. Spalding, S. C., \& Sternberg Jr, P. A. U. L. (1990). Controversies in the management of posterior segment ocular trauma. Retina (Philadelphia, Pa.), 10, S76-82.

19. Weidekamm, C., Köller, M., Weber, M., \& Kainberger, F. (2003). Diagnostic value of highresolution B-mode and doppler sonography for imaging of hand and finger joints in rheumatoid arthritis. Arthritis \& Rheumatism, 48(2), 325-333.

20. Merkel, D., Stahlheber, H., Chupina, V., \& Schneider, C. (2018). Comparison of the quality of
B-scan ultrasound in modern high-end devices. Zeitschrift für Gastroenterologie, 56(12), 1491-1498.

21. Iveković, R., Lovrencić-Huzjan, A., Mandić, Z., \& Talan-Hranilović, J. (2000). Color Doppler flow imaging of ocular tumors. Croatian medical journal, 41(1), 72-75.

22. Abu-Zidan, F. M., Hefny, A. F., \& Corr, P. (2011). Clinical ultrasound physics. Journal of Emergencies, Trauma and Shock, 4(4), 501.

23. Carovac, A., Smajlovic, F., \& Junuzovic, D. (2011). Application of ultrasound in medicine. Acta Informatica Medica, 19(3), 168.

24. Bergès, O., Puech, M., Assouline, M., Letenneur, L., \& Gastellu-Etchegorry, M. (1998). B-modeguided vector-A-mode versus A-mode biometry to determine axial length and intraocular lens power. Journal of Cataract \& Refractive Surgery, 24(4), 529-535.

25. Amstutz, C., Caversaccio, M., Kowal, J., Bächler, R., Nolte, L. P., Häusler, R., \& Styner, M. (2003). A-mode ultrasound-based registration in computeraided surgery of the skull. Archives of otolaryngology-head \& neck surgery, 129(12), 1310-1316.

26. Chang, J. H., Yen, J. T., \& Shung, K. K. (2008). High-speed digital scan converter for highfrequency ultrasound sector scanners. Ultrasonics, 48(5), 444-452.

27. Jakovljevic, M., Hsieh, S., Ali, R., Chau Loo Kung, G., Hyun, D., \& Dahl, J. J. (2018). Local speed of sound estimation in tissue using pulse-echo ultrasound: Model-based approach. The Journal of the Acoustical Society of America, 144(1), 254-266.

28. Burckhardt, C. B. (1978). Speckle in ultrasound Bmode scans. IEEE Transactions on Sonics and ultrasonics, 25(1), 1-6.

29. Park, R., Nyland, T., Lattimer, J., Miller, C., \& Lebel, J. (1981). B-mode gray-scale ultrasound: imaging artifacts and interpretation principles. Veterinary Radiology, 22(5), 204-210.

30. Yeom, E., Nam, K. H., Paeng, D. G., \& Lee, S. J. (2014). Improvement of ultrasound speckle image velocimetry using image enhancement techniques. Ultrasonics, 54(1), 205-216.

31. Figure 2.14. High-frequency $(50 \mathrm{MHz})$ scan of anterior segment produced using an arc-scan. geometry. (2005). Ultrasonography of the eye, by D. Jackson Coleman, MD, FACS, 2005, LWW; Second edition; 35.

32. Figure 2.18. Dislocated hypermature cataractous lens in an eye with vitreous hemorrhage and total retinal detachment. (2005). Ultrasonography of the eye, by D. Jackson Coleman, MD, FACS, 2005, LWW; Second edition; 37

Cite This Article: Nazeeha Waseem et al (2021). B-Mode Ultrasound Diagnosis in Ocular Trauma and its Accuracy. EAS J Radiol Imaging Technol, 3(2), 36-41. 